\title{
Osteoporosis Changes Collagen/Apatite Orientation and Young's Modulus in Vertebral Cortical Bone of Rat
}

\author{
Ryosuke Ozasa ${ }^{1} \cdot$ Takuya Ishimoto $^{1}$ - Sayaka Miyabe ${ }^{1} \cdot$ Jun Hashimoto $^{2} \cdot$ Makoto Hirao $^{3} \cdot$ Hideki Yoshikawa $^{3}$. \\ Takayoshi Nakano ${ }^{1}$
}

Received: 28 September 2018 / Accepted: 17 December 2018 / Published online: 26 December 2018

(c) The Author(s) 2018

\begin{abstract}
This study revealed the distinguished changes of preferential orientation of collagen and apatite and Young's modulus in two different types of osteoporotic bones compared with the normal bone. Little is known about the bone material properties of osteoporotic bones; therefore, we aimed to assess material properties in osteoporotic bones. 66 female Sprague-Dawley rats were used. We analyzed the volumetric bone mineral density, collagen/apatite orientation, and Young's modulus of fifth lumbar vertebral cortex for osteoporotic rats caused by ovariectomy (OVX), administration of low calcium and phosphate content $(\mathrm{LCaP})$ diet, and their combination $(\mathrm{OVX}+\mathrm{LCaP})$, as well as sham-operated control. Osteocyte conditions were assessed by hematoxylin and eosin and immunohistochemical (matrix extracellular phosphoglycoprotein (MEPE) and dentin matrix protein 1 (DMP1)) staining. All osteoporotic animals showed bone loss compared with the sham-operated control. OVX improved craniocaudal Young's modulus by enhancing collagen/apatite orientation along the craniocaudal axis, likely in response to the elevated stress due to osteoporotic bone loss. Conversely, LCaP-fed animals showed either significant bone loss or degraded collagen/apatite orientation and Young's modulus. Osteocytes in LCaP and OVX + LCaP groups showed atypical appearance and MEPE- and DMP1-negative phenotype, whereas those in the OVX group showed similarity with osteocytes in the control group. This suggests that osteocytes are possibly involved in the osteoporotic changes in collagen/ apatite orientation and Young's modulus. This study is the first to demonstrate that osteoporosis changes collagen/apatite orientation and Young's modulus in an opposite manner depending on the cause of osteoporosis in spite of common bone loss.
\end{abstract}

Keywords Osteoporosis $\cdot$ Vertebral bone $\cdot$ Young's modulus $\cdot$ Collagen/apatite orientation $\cdot$ Osteocyte

\section{Introduction}

Osteoporosis is the most common skeletal disorder, which is characterized by low bone mass, resulting in fragile bones with an increased risk of fractures. The most common site of osteoporotic fractures is the spine. From a mechanical

Takayoshi Nakano

nakano@mat.eng.osaka-u.ac.jp

1 Division of Materials and Manufacturing Science, Graduate School of Engineering, Osaka University, 2-1 Yamada-oka, Suita, Osaka 565-0871, Japan

2 Department of Rheumatology, National Hospital Organization, Osaka Minami Medical Center, 2-1 Kidohigashi, Kawachinagano, Osaka 586-8521, Japan

3 Department of Orthopaedic Surgery, Graduate School of Medicine, Osaka University, 2-2 Yamada-oka, Suita, Osaka 565-0871, Japan viewpoint, vertebral fractures must be caused either by reduced strength of the vertebral bodies or by increased stress [1] due to the reduced bone mass.

The bone is responsive to changes in mechanical stimuli. As has been well documented and generally recognized, the bone increases its mass in response to elevated stress [2]. However, despite the elevated in vivo stress, bone loss is inevitable under osteoporotic conditions, which substantially means inability of adaptation by bone mass gain. Clarifying whether bone adaptation under osteoporotic conditions is accomplished via mechanisms other than bone mass change is important to understand biomechanics during osteoporosis progression. This might partly explain the dissociation between dual-energy X-ray absorptiometry (DXA)-measured bone mineral density (BMD) and bone fragility (fracture risk) $[3,4]$. In osteoporotic conditions, the trabecular bone is known to increase its structural anisotropy because more horizontal (secondary) trabeculae are lost than vertical 
(primary) trabeculae [1], resulting in more aligned trabeculae in the craniocaudal axis along which the principal load is applied [5]. This would compensate, to some extent, for negative effects of bone loss and maintain strength against craniocaudally loaded stress [1]. The hypothesis in this study was that cortical bone also has the ability to compensate for bone loss by enhancing the mechanical property along the craniocaudal axis, the principally loaded direction, to meet the increased mechanical demands caused by osteoporotic bone loss. This requires the alteration of bone material anisotropy.

The focus of this study was on the preferential orientation of collagen and biological apatite, i.e., nanometer-scale structural anisotropy [6] that has been proven to be one of the important determinants of bone material integrity $[7,8]$. In normal mineralization, the crystallographic $c$-axis of apatite aligns almost parallel to the collagen fiber direction via an epitaxial apatite crystallization on the collagen template [9], resulting in the formation of an oriented nano-composite. Hence, the stronger direction of apatite [10] and collagen [11] co-aligns in one direction, which makes the bone stiff and tough in the collagen/apatite-oriented direction without the change in the bone mass or BMD.

In this study, different types of osteoporotic rat models were prepared by ovariectomy (OVX), administration of low calcium and phosphate content (LCaP) diet, and their combination, which are well-accepted animal models for osteoporosis. Material properties including Young's modulus as an index of resistance to mechanical stress, the preferential orientation of collagen/apatite, and a volumetric BMD (vBMD) were analyzed in the cortical bone of the lumbar vertebral body to investigate adaptive responses in the cortical bone material under osteoporotic conditions with increased stress along the craniocaudal direction. The biological conditions of osteocytes were additionally assessed because osteocytes are believed to regulate bone adaptive response [12] and may be involved in variations of bone material properties under osteoporosis.

\section{Materials and Methods}

\section{Study Design}

Sample size calculations were performed using power and sample size calculation software. Since our key foci in this study were Young's modulus and preferential collagen/apatite orientation, the calculations were performed based on pre-published information $[8,13]$ regarding these parameters measured by the same apparatus. It was estimated that 4 and 6 animals for Young's modulus and orientation, respectively, are required to achieve $95 \%$ confidence interval and a power of $80 \%$; therefore, we determined to use 6 animals per group.

This study used 66 5-week-old female Sprague-Dawley rats (Japan SLC, Inc., Japan). They were kept in conventional condition and individually housed in plastic cages at $25^{\circ} \mathrm{C}$ and $55 \%$ humidity with a 12-h light/12-h dark cycle throughout the study. The rats were fed ad libitum with a regular diet, which was composed of $1.25 \%$ calcium and 0.90\% phosphate (CRF-1; Charles River Japan Inc., Japan) and tap water for 1 week of acclimation. According to previous studies [14, 15], 6-week-old animals were randomly allocated into 11 groups $(n=6)$; six animals were sacrificed to prepare a baseline group with the remainder assigned to either the OVX- or sham-operated control groups. Operations were performed under anesthesia with intraperitoneal administration of sodium pentobarbital. Following the operations, the rats were fed with either the regular diet or a calcium- and phosphate-deficient diet containing $0.03 \%$ calcium and $0.30 \%$ phosphate (CLEA Diet No.011; CLEA Japan. Inc., Japan). The rats were assigned to the following groups: (1) sham-operated, fed with the regular diet (control group); (2) OVX-operated, fed with the regular diet (OVX group); (3) sham-operated, fed with the LCaP diet (LCaP group); and (4) OVX-operated, fed with the LCaP diet (OVX + LCaP group). Previous studies have reported that a 3-month period after operation is sufficient to induce bone loss in rats fed with calcium-deficient diet [14], but it was inadequate in OVX-operated rats $[16,17]$. Therefore, the control and OVX animals were sacrificed by an intravenously administered overdose of sodium pentobarbital at 1,3 , and 6 months postoperatively, and the $\mathrm{LCaP}$ and $\mathrm{OVX}+\mathrm{LCaP}$ animals were sacrificed at 1 and 3 months postoperatively. Herein, the periods of 1,3 , and 6 months are referred to as $1 \mathrm{M}, 3 \mathrm{M}$, and $6 \mathrm{M}$, respectively. Immediately before euthanasia, body weights were measured. All animal procedures and protocols were approved by the Animal Experiment Committee of Osaka University Graduate School of Engineering (approval number: 4). The analyses were done unblinded.

\section{Sample Preparation}

The fifth lumbar (L5) vertebral bone was extracted from the animals immediately after sacrifice, immersed in a $10 \mathrm{wt} \%$ neutral buffered formalin solution for 1 week, and immersed in a 70\% ethanol until analysis. After analysis via peripheral quantitative computed tomography (pQCT), the central part of the L5 vertebral bone was cut perpendicular to the craniocaudal axis of the vertebra. The cross section of the specimen was ground by a series of emery paper with increasing fineness (\#600, \#800, \#1200, \#1600, and \#2000) under deionized water for a microbeam X-ray diffraction ( $\mu$-XRD) analysis, followed by mirror polished 
using a microcloth (Buehler Ltd., Lake Bluff, IL, USA) with up to $0.05-\mu \mathrm{m}$ alumina suspension for nanoindentation measurement. The anterior cortical region of the vertebral body was used for all analyses because the anterior cortex was reported to be a major load-bearing site for axial compression or flexion [18].

\section{vBMD and Bone Area Measurement by PQCT and Estimation of Relative Stress Applied to the Vertebral Body}

vBMD and bone area of the L5 vertebral bone was measured using pQCT system (XCT Research SA+; Stratec Medizintechnik GmbH, Birkenfeld, Germany). The central cross section of the L5 vertebra along with the craniocaudal axis was scanned with a resolution of $80 \times 80 \times 460 \mu^{3}$. Bone tissue was defined above a threshold value of $267 \mathrm{mg} / \mathrm{cm}^{3}$ [19]. vBMD values were analyzed in the three regions with $240 \times 240 \mu \mathrm{m}^{2}$ at which the $\mu$-XRD analyses were performed. The bone area was determined by counting voxels with the vBMD of $\geq 267 \mathrm{mg} /$ $\mathrm{cm}^{3}$ (see Fig. 1). Relative stress applied to the vertebral body was estimated as the ratio of body weight/bone area.

\section{Analysis of Preferential Orientation of Apatite c-Axis Using $\mu$-XRD}

To assess the degree of preferential orientation of apatite $c$-axis of L5 vertebral bone along the craniocaudal axis of the vertebra, X-ray diffraction analysis was performed using the $\mu$-XRD system (M18XHF ${ }^{22}$-SR, Mac Science Co., Yokohama, Japan). The detailed conditions for $\mu$-XRD were described in the previous paper $[6,7]$. The incident beam was focused into a beam spot of $50 \mu \mathrm{m}$ in diameter by a metal collimator. The scattered diffraction was counted for $4000 \mathrm{~s}$ to obtain adequate diffraction intensity. The two representative diffraction peaks for apatite, (002) and (310), appearing at Bragg angles of 25.9 and 39.8 degrees, respectively, were detected by swinging and rotating the specimen in an appropriate range. Thus, diffraction peaks were obtained from an irradiated area of $\sim 250 \mu \mathrm{m}$ in diameter on the specimen surface. The degree of preferential orientation of apatite $c$-axis was determined as an intensity
Fig. 1 Bone densitometric analysis. a Typical pQCT images of L5 vertebral body in each group at $3 \mathrm{M}$ and $6 \mathrm{M}$ and b variation of vBMD measured within anterior cortical bone. $* * p<0.01 ; * p<0.05 . v B M D$ volumetric bone mineral density a

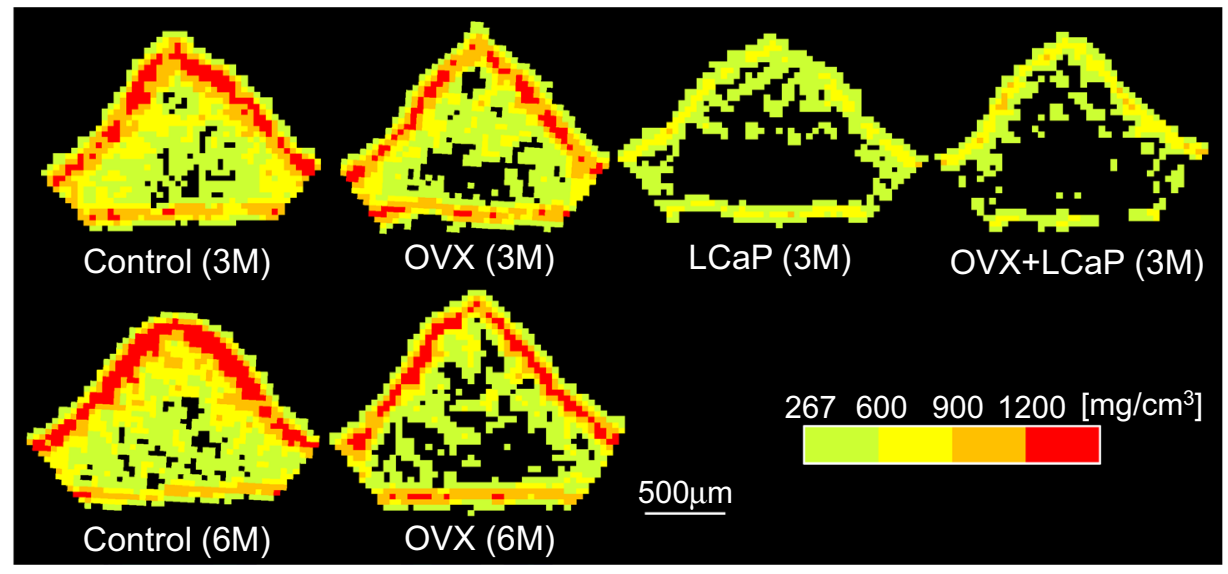

b

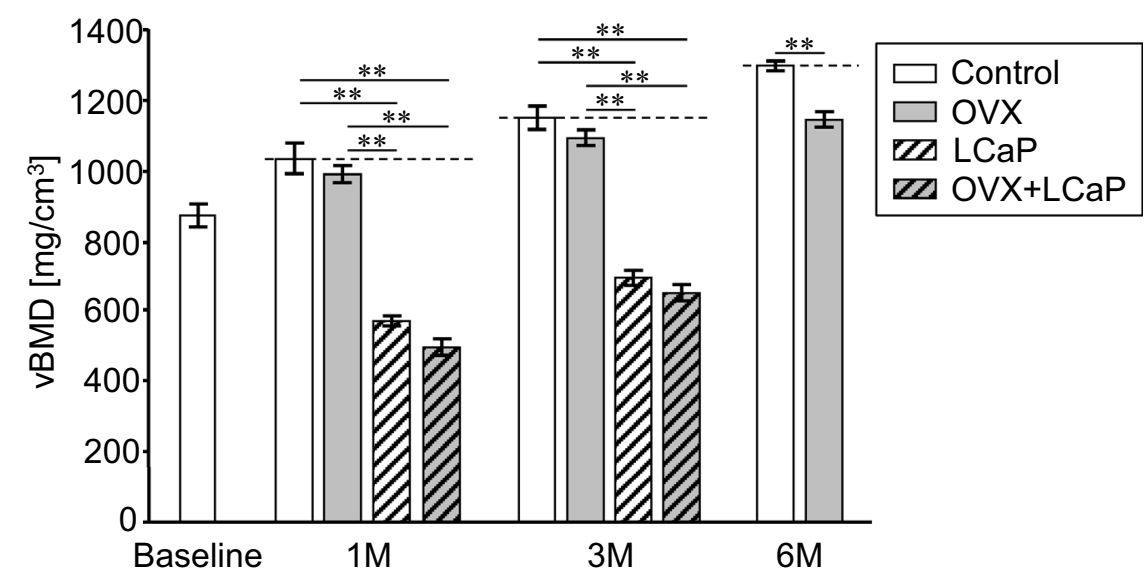


a

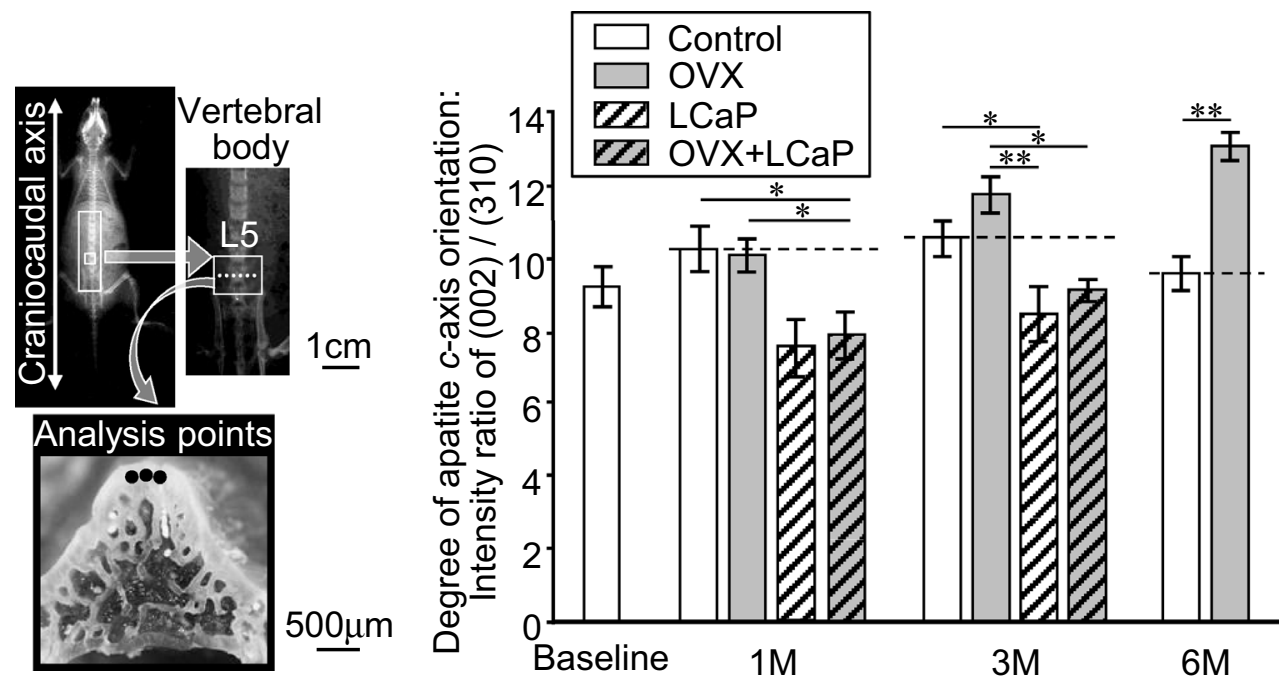

b


Control (6M)

C

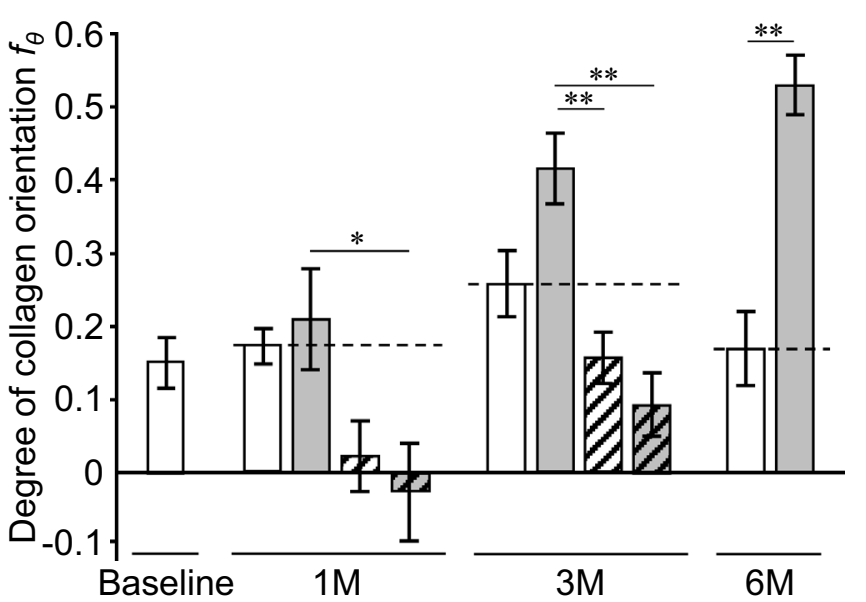

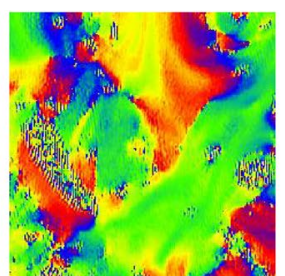

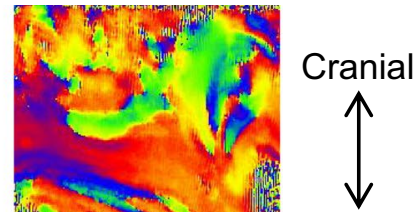

Caudal

$$
\text { LCaP (3M) OVX+LCaP (3M) }
$$

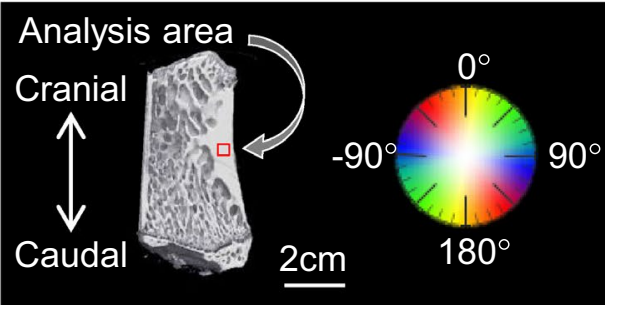

d

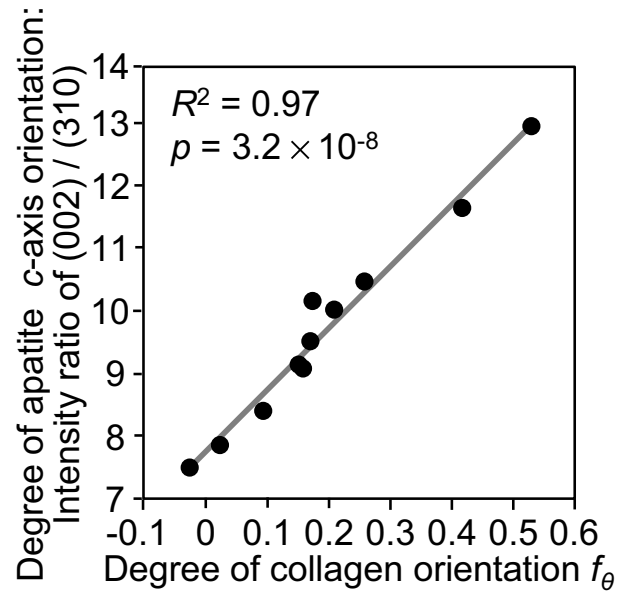


4Fig. 2 Preferential orientation of the apatite $c$-axis and collagen along the craniocaudal axis analyzed within the anterior cortical bone of the L5 vertebral body. a Degree of apatite $c$-axis orientation. The higher value represents the more prominent orientation along the craniocaudal axis. b Birefringence images with colors, which indicate the slowaxis direction of birefringence representing the preferential collagen orientation direction and $\mathbf{c}$ quantitative degree of the collagen orientation. d Correlation between the degree of apatite orientation and that of collagen orientation. ${ }^{*} p<0.01 ; * p<0.05$

ratio of $(002) /(310)[6,7]$. Randomly orientated apatite (hydroxyapatite) powders (NIST \#2910) represent the value of 2 in this XRD system [6]; thus, the value $>2$ represents the preferential orientation in the analyzed direction. Measurements were performed at three points within the cortical bone as indicated in Fig. 2a, and the data were averaged.

\section{Analysis of Collagen Orientation by Birefringence Method}

To evaluate the degree of preferential alignment of collagen of L5 vertebral bone along the craniocaudal axis of the vertebra, the bone specimens after nanoindentation measurement were decalcified, embedded into paraffin, and parallel cut to the craniocaudal axis into $4-\mu \mathrm{m}$-thick sections. Deparaffinized sections were observed by a two-dimensional birefringence measurement system (WPA-micro; Photonic Lattice, Miyagi, Japan) attached to an upright microscope (BX60; Olympus, Tokyo, Japan). Birefringence analysis of collagen was performed using a WPA-VIEW software (version 2.4.2.9; Photonic Lattice) as previously described [20]. For quantitative comparison of collagen orientation, the orientation order parameter $f_{\theta}$ was calculated based on the angle distribution of collagen against the craniocaudal axis as previously described [21]. $f_{\theta}$ takes a value ranging from -1 (collagen perfectly aligned perpendicular to the craniocaudal direction of vertebra bone) to 1 (collagen perfectly aligned parallel to the craniocaudal direction of vertebra bone).

\section{Analysis of Young's Modulus by Nanoindentation}

The bone specimens were fully dried with a graded ethanol series (70-100\%), fixed on a specimen holder, and kept in $25{ }^{\circ} \mathrm{C}$ for $24 \mathrm{~h}$ for thermal stabilization prior to nanoindentation test. Young's modulus of L5 vertebral bone was measured along the craniocaudal axis of the vertebra using a nanoindentation system (ENT-1100a; Elionix, Tokyo, Japan) with a Berkovich diamond indenter. Load-depth measurements were carried out according to the established condition [22]. The loading/unloading rate and maximum load were $400 \mu \mathrm{N} / \mathrm{s}$ and $6 \mathrm{mN}$, respectively. To minimize the effects of the viscoelastic behavior of the bone, a constant maximum load was held for $180 \mathrm{~s}$ before unloading, as determined previously [22]. All measurements also included a second constant load held for $30 \mathrm{~s}$ at $10 \%$ of the maximum load to establish the thermal drift rate and correct the data. As a result of the loading/unloading scheme, indents with a width of less than $10 \mu \mathrm{m}$ were created. Young's modulus was determined by the method of Oliver and Pharr [23]. Poisson's ratio of bone was assumed to be 0.3 , and the region between 95 and $50 \%$ of the maximum load was used to calculate the slope of the unloading curve. Five indentations were created with an interval of $125 \mu \mathrm{m}$ on the anterior cortex region in which the $\mu$-XRD analysis and vBMD measurements were performed, and the results were averaged.

\section{Evaluation of Biological Conditions of Osteocytes by Hematoxylin and Eosin (HE) and Immunohistochemical Staining}

The fourth lumbar (L4) vertebral bone was extracted from rats immediately after sacrifice and immersed in $4 \%$ paraformaldehyde at $4{ }^{\circ} \mathrm{C}$. After decalcification with a $0.5 \mathrm{M}$ EDTA-2Na solution (pH 7.4) for 7 days at $4{ }^{\circ} \mathrm{C}$, specimens were dehydrated with a graded ethanol series, embedded in paraffin, and perpendicularly cut to the craniocaudal axis into 4- $\mu \mathrm{m}$-thick sections. On the basis of osteocyte morphology observed under HE staining, normal and atypical osteocytes were determined according to Bentolila et al. [24]. Immunohistochemical staining for matrix extracellular phosphoglycoprotein (MEPE) [25] and dentin matrix protein 1 (DMP1) [26] were performed at $3 \mathrm{M}$ as described in previous studies $[27,28]$ to evaluate normality of osteocytes.

\section{Statistical Analysis}

In all quantitative data measurements, 6 animals were used and the data were averaged and shown as mean \pm standard error (SE). Statistical comparisons between the two means were performed using two-tailed unpaired student's $t$ test subsequently to F-test for homoscedasticity. Comparisons among four means were done by one-way analysis of variance. Post hoc Tukey HSD comparisons or Games-Howell comparisons were conducted, according to test for homoscedasticity. Single and multiple regression analyses were performed to determine contributory factors to Young's modulus. For detecting multicollinearity prior to multiple regression analysis, tolerance and variance inflation factors (VIF) were calculated as follows:

Tolerance $=1-R_{j}^{2}$

$\mathrm{VIF}=1 /$ Tolerance

where $R_{j}^{2}$ is the coefficient of determination of a regression of explanator $j$ on all other explanators. A tolerance $(<0.10)$ 
and a VIF $(\geq 10)$ were considered to indicate a multicollinearity problem [29]. A $p$ value of $<0.05$ was considered statistically significant. SPSS version $14.0 \mathrm{~J}$ software (SPSS Japan Inc., Japan) for microsoft windows was used for the statistical analyses.

\section{Results}

\section{Body Weight, Area of L5 Vertebra, and Estimated Relative Stress in Osteoporotic Rats}

The control animals showed normal weight prior to (baseline) and posterior to treatment (1M, 3M, and $6 \mathrm{M})$ (Table 1), which deviated only by at most $6 \%$ from the weight curve that the animal supplier provided. As shown in Table 1, body weight increased in the OVX group but decreased in the LCaP and OVX $+\mathrm{LCaP}$ groups compared with the control group. OVX increases body weight by increasing adipose tissue [30]. The L5 vertebral body area decreased in the OVX group and further decreased in the LCaP and OVX + LCaP groups (Table 1). Therefore, OVX mildly advanced the osteoporotic phenotype, but LCaP diet administration acutely did. As a result, the estimated stress on L5 vertebral body significantly increased in all osteoporotic groups than in the control group.

\section{vBMD of L5 Vertebral Cortex in Osteoporotic Rats}

Figure 1 shows the vBMDs of osteoporotic bones. The OVX group had a gradual vBMD decrease and significantly lower vBMD at $6 \mathrm{M}$ than the control group. The $\mathrm{LCaP}$ and OVX + LCaP groups had drastic vBMD decline and significantly lower vBMD than the control group throughout the experimental periods. No difference was observed between the $\mathrm{LCaP}$ and $\mathrm{OVX}+\mathrm{LCaP}$ groups.

\section{Preferential Orientation of Collagen/Apatite Along the Craniocaudal Axis of the Vertebral Cortex}

Figure 2a shows the preferential orientation of apatite $c$-axis of osteoporotic vertebral bones analyzed along the craniocaudal axis. Unlike the vBMD, the apatite orientation changed in the opposite manner between the OVX group and $\mathrm{LCaP}$ and $\mathrm{OVX}+\mathrm{LCaP}$ groups. The OVX group showed gradual upward tendency, while the $\mathrm{LCaP}$ and $\mathrm{OVX}+\mathrm{LCaP}$ groups showed a clear drop. The degree of collagen orientation showed similar tendency with the apatite orientation as shown in Fig. 2b, c. The OVX group showed more homogenous collagen directionality as indicated by the birefringence color map (Fig. 2b) and higher degree of collagen orientation along the craniocaudal direction, while the LCaP and OVX $+\mathrm{LCaP}$ groups demonstrated heterogeneous collagen 


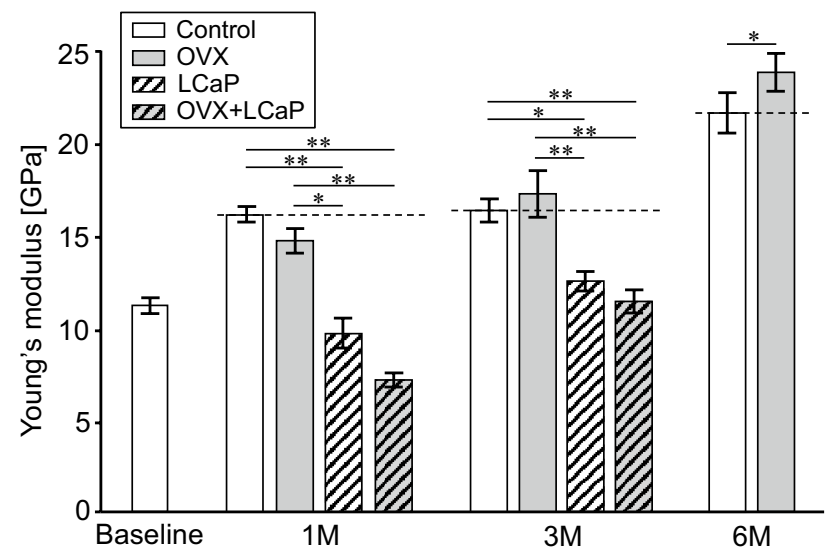

Fig. 3 Young's modulus measured along the craniocaudal axis of the L5 vertebral body cortex. ${ }^{* *} p<0.01 ; * p<0.05$

directionality (multicolor in birefringence images) and lower degree of collagen orientation. The degree of preferential apatite $c$-axis orientation strongly and positively correlated with that of collagen orientation $\left(R^{2}=0.97, p=3.2 \times 10^{-8}\right)$ (Fig. 2d). These results indicate that the nanometer-scale bone material anisotropy along with the craniocaudal axis was enhanced in the OVX group and, by contrast, seriously degraded in the $\mathrm{LCaP}$ and OVX $+\mathrm{LCaP}$ groups.

\section{Young's Modulus Along the Craniocaudal Axis of the Vertebral Cortex}

Figure 3 shows Young's modulus of osteoporotic bones analyzed along the craniocaudal axis of the vertebra. The modulus in the OVX group tended to gradually increase compared to the control group. At $6 \mathrm{M}$, the modulus in the OVX group was significantly higher than the control despite the significantly decreased vBMD. The moduli in the LCaP and $\mathrm{OVX}+\mathrm{LCaP}$ groups significantly decreased throughout the experiment.

\section{Contributions of vBMD and the Collagen/Apatite Orientation to Young's Modulus}

From the single regression analysis, all material properties of vBMD $\left(R^{2}=0.78, p=3.1 \times 10^{-4}\right)$, apatite orientation $\left(R^{2}=0.76, p=4.5 \times 10^{-4}\right)$, and collagen orientation $\left(R^{2}=0.71, p=1.1 \times 10^{-3}\right)$ significantly and positively correlated with Young's modulus. In this study, the degree of apatite $c$-axis orientation and that of collagen orientation showed multicollinearity (Tolerance $=0.03<0.1$, VIF $=30.97>10$ ), whereas no multicollinearity was found between collagen orientation and vBMD (Tolerance $=0.54>0.1, \mathrm{VIF}=1.85<10)$ and apatite $c$-axis orientation and $\mathrm{vBMD}$ (Tolerance $=0.44>0.1, \mathrm{VIF}=2.24<10$ ); therefore, collagen orientation was excluded as explanatory variables for multiple regression analysis to Young's modulus. Multiple regression analysis revealed that the preferential orientation of apatite makes comparable and significant contributions with vBMD to Young's modulus as represented by the similar $\beta$ (Table 2 ).

\section{Relationship Between Material Properties and the Estimated Relative Stress}

Figure 4 shows the relationship between material properties (Young's modulus along the craniocaudal axis, vBMD, and degree of apatite orientation along the craniocaudal axis) and the estimated relative stress. Since the orientations of apatite and collagen showed strong correlation (Fig. 2d), the result with collagen orientation was not shown. Young's modulus in the control and OVX groups showed positive relations with stress and age (Fig. 4a). vBMD in the control and OVX groups showed positive relation, but the slope was extremely small in the OVX group (Fig. 4b). The degree of apatite orientation in the control group was hardly changed, but that in OVX group showed positive relation (Fig. 4c). $\mathrm{LCaP}$ and $\mathrm{OVX}+\mathrm{LCaP}$ groups deteriorated bone material properties even if applied stress was estimated to be higher than that in the control group.

\section{Changes in Osteocyte Morphology and MEPE/DMP1 Expression}

Figure 5 shows the osteocyte conditions estimated by HE and immunohistochemical staining. HE staining revealed that the ratio of atypical osteocyte increased in the LCaP and OVX $+\mathrm{LCaP}$ groups, whereas no significant increase was observed in the OVX group compared to the control group (Fig. 5a, b). MEPE and DMP1 were positively expressed either in the control or OVX group, while neither was expressed in the $\mathrm{LCaP}$ nor in the $\mathrm{OVX}+\mathrm{LCaP}$ groups (Fig. 5c).

\section{Discussion}

In this study, the vBMD, the preferential orientation of collagen/apatite, and Young's modulus of L5 vertebral cortex for the osteoporotic (OVX, LCaP, and $\mathrm{OVX}+\mathrm{LCaP})$ and normal (control) rats were analyzed. The collagen/apatite

Table 2 Relative contribution of vBMD and apatite orientation to Young's modulus determined by multiple regression analysis

\begin{tabular}{lllll}
\hline vBMD & & \multicolumn{2}{l}{$\begin{array}{l}\text { Apatite orien- } \\
\text { tation }\end{array}$} \\
\cline { 1 - 2 } \cline { 5 - 6 }$\beta$ & $p$ & & $\beta$ & $p$ \\
\hline 0.51 & 0.032 & & 0.47 & 0.047 \\
\hline
\end{tabular}



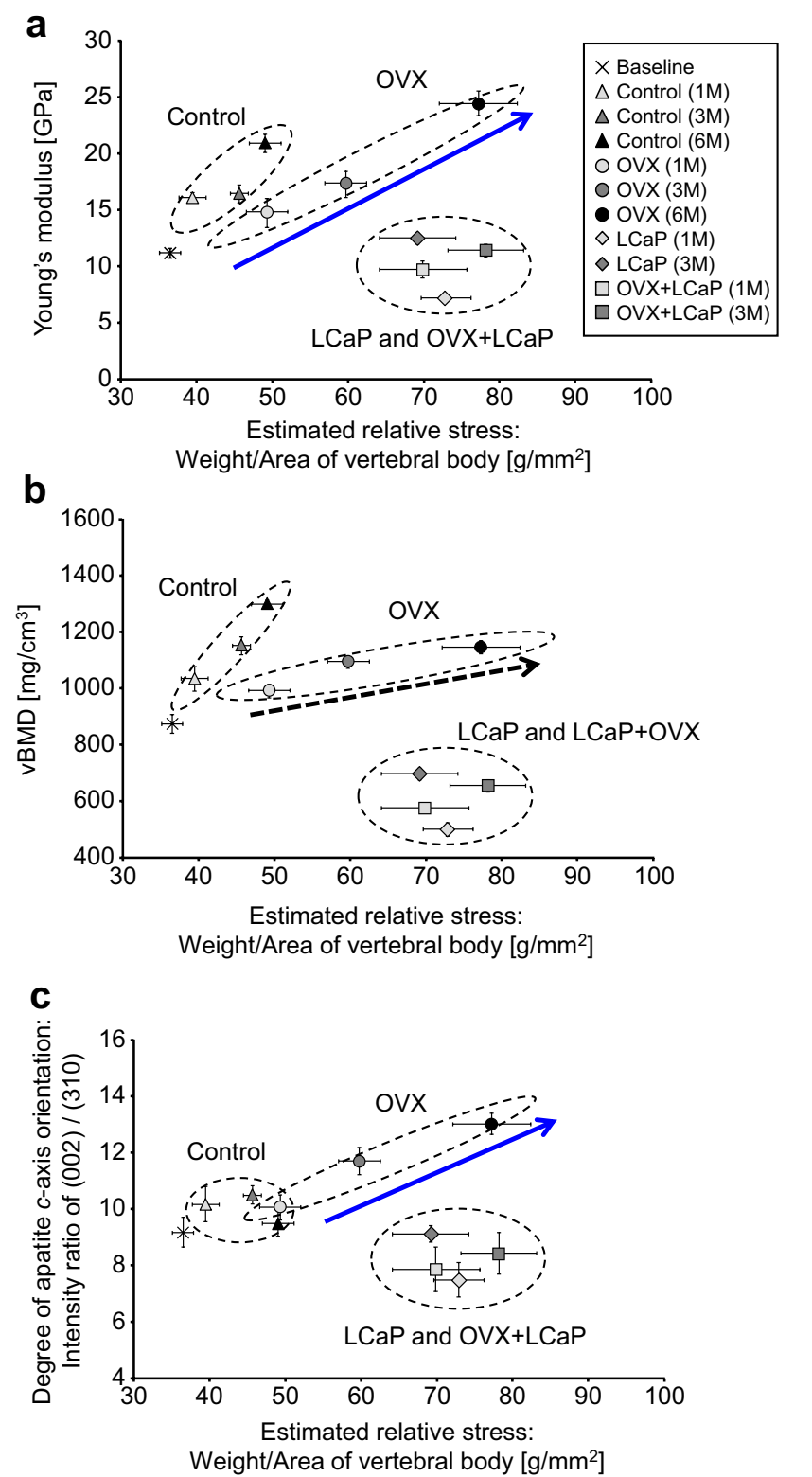

Fig. 4 Variations in bone material properties as a function of the estimated relative stress. Relationship between a Young's modulus, b vBMD, and $\mathbf{c}$ degree of apatite $c$-axis orientation with the estimated relative stress

orientation and Young's modulus were measured along the craniocaudal axis in which principal stress is applied [5]. Our results clearly demonstrated the gap in bone material phenotype depending on osteoporotic conditions between the OVX group, and $\mathrm{LCaP}$ and $\mathrm{OVX}+\mathrm{LCaP}$ groups. Because of the strong effect of insufficient intake of calcium and phosphate, the $\mathrm{LCaP}$ and $\mathrm{OVX}+\mathrm{LCaP}$ groups denoted almost the same tendency in all material properties.

Our hypothesis was that vertebral cortical bone increases its material property along the craniocaudal axis, the principally loaded direction, to compensate for bone loss that is inevitable under osteoporotic conditions; therefore, we used young animals that show rapid gain of body weight. Bone loss occurred in all osteoporotic models in comparison to the control group; therefore, stress applied to the vertebral body can be estimated to increase. Young's modulus in the OVX groups at $6 \mathrm{M}$ exceeded the age-matched modulus, representing that the mechanical property in bone material adaptively changed to the elevated stress under OVX-induced osteoporotic condition. This is largely attributed to the significantly enhanced collagen/apatite orientation because vBMD in the OVX group was significantly lower than that in the control group at $6 \mathrm{M}$. In contrast, the moduli and collagen/apatite orientation in the $\mathrm{LCaP}$ and $\mathrm{OVX}+\mathrm{LCaP}$ groups significantly degraded. These results demonstrate that osteoporosis changes collagen/apatite orientation and Young's modulus in an opposite manner depending on the cause of osteoporosis, which was revealed for the first time in this study. We used 6-week-old animals; therefore, the variations of material properties investigated in this study include the effects of skeletal growth, which cannot be eliminated. Although using skeletally mature animals would be better to minimize the effect of growth, we had put priority on the weight gain in this study. However, the result would indicate that growth had little influence on the increase in collagen/apatite orientation in the OVX group and its drop in the $\mathrm{LCaP}$ and $\mathrm{OVX}+\mathrm{LCaP}$ groups, because the degree of orientation was kept unchanged in the control group throughout the study. The enhancement of apatite orientation along the craniocaudal axis following OVX operation was reported previously using the 11-month-old mature rat (OVX for 6 months) [13] and the 9-17-year-old monkey (OVX for 17 months) [31]. Thus, the collagen/apatite orientation (nanometer-scale anisotropy) is assumed to become prominent under estrogen-deficient condition independent of animal species (primate or rodent) and ages.

Morphological and immunohistochemical observations indicate that osteocytes in the $\mathrm{LCaP}$ and $\mathrm{OVX}+\mathrm{LCaP}$ groups were not in a normal condition, while those in OVX group were less affected. The osteocyte conditions estimated in this study appear to associate with the changes in Young's modulus and orientation along with principally loaded direction. Moreover, the osteocyte network including lacuno-canalicular architecture was also proposed to be important for organization of anisotropic bone matrix [20]. However, whether and how these osteocyte conditions affect the variations of material properties remains unclear, which needs further investigations.

In terms of the osteocyte abnormality estimated in the rats fed with the LCaP diet, the contents of calcium and phosphate in the diet used are extremely low. Low dietary calcium causes a decrease in serum calcium, triggering the release of parathyroid hormone $(\mathrm{PTH})$ from the parathyroid gland [32]. Excessive PTH binding to PTH receptor on cell 
Fig. 5 Normality of osteocytes. a Definition of normal $(\mathrm{N})$ and several conditions of atypical osteocytes (S: lacuna with shrunken osteocyte, E: empty lacunae, P: osteocyte with pyknotic nuclei, $\mathrm{H}$ : osteocyte with hollow nuclei) observed under HE staining [24], and $\mathbf{b}$ the ratio of atypical osteocytes. c Immunohistochemically stained images with MEPE and DMP1 at $3 \mathrm{M}$. Arrowheads indicate osteocytes positive for MEPE or DMP1. $* * p<0.01 ; * p<0.05$ a



b



C

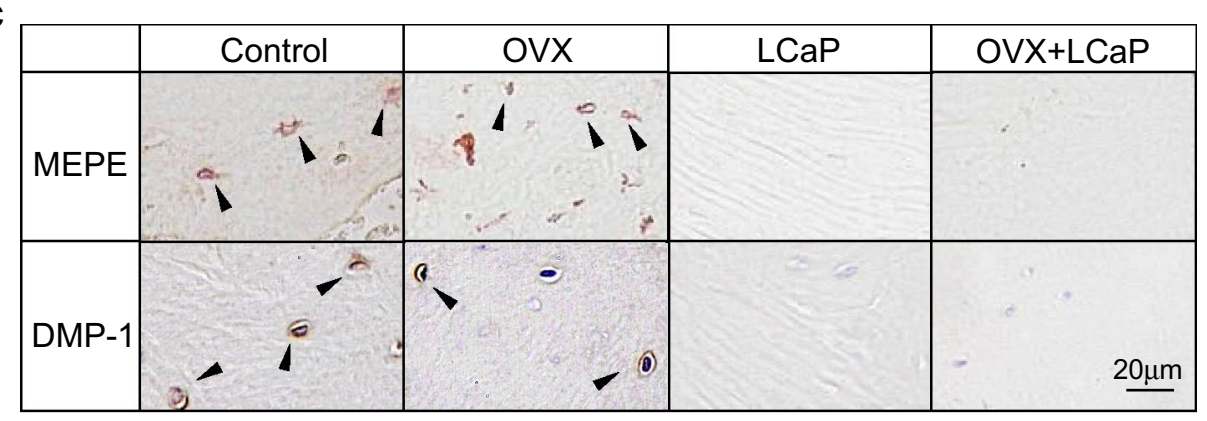

membrane induces apoptosis of osteocyte [33]. Additionally, phosphate depletion also induces abnormalities in osteocytes [34]. Moreover, the dietary $\mathrm{Ca} / \mathrm{P}$ ratio is reported to associate with modification of PTH concentration $[35,36]$. Thus, the content and $\mathrm{Ca} / \mathrm{P}$ ratio, but which were not modified in this study, might vary the result of adaptive responses. This is a major limitation of this study. Future studies clarifying the dose-dependent effect and mechanisms through which osteocyte regulates collagen/apatite orientation and Young's modulus under estrogen deficiency and calcium and phosphate deficiency-induced conditions from genetic, molecular, and cellular aspects should be conducted.

Bone strength or fracture resistance is determined by both material and structural properties [37]. As the focus in this study was material properties, whole bone mechanical property was not determined. According to the literature, the OVX-operated rats lost their vertebral strength [13, 38, 39], which must be due to the significant bone loss (structural property). The increase in craniocaudal Young's modulus in the OVX group in this study would be the result of adaptive response through which the bone managed to try not to reduce bone strength even when bone loss is inevitable.

Clinical studies claimed that the DXA-BMD is not well associated with vertebral fracture risk of osteoporotic and diabetic patients $[3,4]$. Based on the literature, BMD predicts approximately only $50-80 \%$ of the variation in bone fragility [40], because it cannot detect preferential collagen/ apatite orientation, which also influences bone strength. The specific nature of preferential collagen/apatite orientation is its ability to express nanometer-scale anisotropy of bone, unlike other indices characterizing bone material property such as vBMD, degree of mineralization [41], thickness and crystallinity of apatite mineral [42], collagen cross-linkage [43], which are important for bone strength but do not depict anisotropic features of the bone. Considering collagen/apatite orientation is believed to be beneficial in the diagnosis and treatment of bone diseases. However, that the enhanced mechanical property in a certain direction would degrade in other directions, likely resulting in higher fracture risk when subjected to uncommon loads, should be considered [1].

In conclusion (Fig. 6), in this study, we analyzed the craniocaudal Young's modulus as a parameter of resistance to mechanical stress as well as vBMD and preferential orientation of collagen/apatite as explanatory factors of Young's modulus in the fifth lumbar vertebral cortex of OVXoperated and/or low calcium and phosphate diet-fed rats, of which both showed osteoporotic phenotype of reduced bone mass. Notably, in the OVX group, Young's modulus along the craniocaudal axis in which the principal stress is applied significantly increased with the elevation of stress, indicating expression of adaptive response through material property. This increase in Young's modulus was well explained by the degree of collagen/apatite orientation that characterizes nanometer-scale anisotropy in bone. This is the first study to demonstrate enhancement of bone mechanical property along with the elevated stress through the modification of anisotropy in collagen/apatite arrangement. In contrast, the LCaP diet-fed rats $(\mathrm{LCaP}$ and $\mathrm{OVX}+\mathrm{LCaP}$ 
Fig. 6 Schematic illustration of two pathological conditions of osteoporotic bones based on collagen/apatite orientation and the normality of osteocytes



groups) showed either significant bone loss or degradation of collagen/apatite orientation. These changes in material properties, especially collagen/apatite orientation, appear to correspond with morphological and immunohistochemical phenotypes of osteocytes; therefore, osteocytes may be possibly involved in the changes in collagen/apatite orientation which occurs under osteoporotic conditions.

Acknowledgements This work was funded by the Grants-in-Aid for Scientific Research (Grant Nos. JP18H05254, JP18H03844, and JP17H06224) from the Japan Society for the Promotion of Science (JSPS).

Author Contributions Study conception and design: TN. Acquisition of data or analyses and interpretation of data: RO, TI, SM, JH, MH, HY, and TN. Drafting the manuscript or revising it critically for important intellectual content: RO, TI, SM, and TN. Approving final version of manuscript: RO, TI, SM, JH, MH, HY, and TN. TN takes responsibility for the integrity of the data analysis.

\section{Compliance with Ethical Standards}

Human and Animal Rights and Informed Consent All procedures and protocols performed in studies were approved by the Animal Experiment Committee of Osaka University Graduate School of Engineering (approval number: 4). This article does not contain any studies with animals performed by any of the authors.

Conflict of interest Ryosuke Ozasa, Takuya Ishimoto, Sayaka Miyabe, Jun Hashimoto, Makoto Hirao, Hideki Yoshikawa, and Takayoshi Nakano declare that they have no conflict of interest. Human and Animal Rights and Informed Consent All procedures and protocols performed in studies were approved by the Animal Experiment Committee of Osaka University Graduate School of Engineering (approval number: 4). This article does not contain any studies with animals performed by any of the authors.
Open Access This article is distributed under the terms of the Creative Commons Attribution 4.0 International License (http://creativeco mmons.org/licenses/by/4.0/), which permits unrestricted use, distribution, and reproduction in any medium, provided you give appropriate credit to the original author(s) and the source, provide a link to the Creative Commons license, and indicate if changes were made.

\section{References}

1. Homminga J, Van-Rietbergena B, Lochmüllerc EM, Weinansd H, Ecksteine F, Huiskes R (2004) The osteoporotic vertebral structure is well adapted to the loads of daily life, but not to infrequent "error" loads. Bone 34:510-516. https://doi.org/10.1016/j. bone.2003.12.001

2. Frost HM (1987) Bone "mass" and the "mechanostat": a proposal. Anat Rec 219:1-9.https://doi.org/10.1002/ar.1092190104

3. Riggs BL, Melton LJ (2002) Bone turnover matters: the raloxifene treatment paradox of dramatic decreases in vertebral fractures without commensurate increases in bone density. J Bone Miner Res 17:11-14. https://doi.org/10.1359/jbmr.2002.17.1.11

4. Yamamoto M, Yamaguchi T, Yamauchi M, Kaji H, Sugimoto T (2009) Diabetic patients have an increased risk of vertebral fractures independent of BMD or diabetic complications. J Bone Miner Res 24:702-709. https://doi.org/10.1359/jbmr.081207

5. Smit TH (2002) The use of a quadruped as an in vivo model for the study of the spine-biomechanical considerations. Eur Spine J 11:137-144. https://doi.org/10.1077/s005860100346

6. Nakano T, Kaibara K, Tabata Y, Nagata N, Enomoto S, Marukawa E, Umakoshi Y (2002) Unique alignment and texture of biological apatite crystallites in typical calcified tissues analyzed by microbeam x-ray diffractometer system. Bone 31:479487. https://doi.org/10.1016/S8756-3282(02)00850-5

7. Ishimoto T, Nakano T. Umakoshi Y, Yamamoto M, Tabata Y (2013) Degree of biological apatite $c$-axis orientation rather than bone mineral density controls mechanical function in bone regenerated using rBMP-2. J Bone Miner Res 28:1170-1179. https://doi.org/10.1002/jbmr.1825

8. Ishimoto T, Sato B, Lee J-W, Nakano T (2017) Co-deteriorations of anisotropic extracellular matrix arrangement and intrinsic mechanical property in c-src deficient osteoporotic 
mouse femur. Bone 103:216-223. https://doi.org/10.1016/j. bone.2017.06.023

9. Landis WJ (1995) The strength of a calcified tissue depends in part on the molecular structure and organization of its constituent mineral crystals in their organic matrix. Bone 16:533-544. https ://doi.org/10.1016/8756-3282(95)00076-P

10. Viswanath B, Raghavan R, Ramamurty U, Ravishankar N (2007) Mechanical properties and anisotropy in hydroxyapatite single crystals. Scr Mater 57:361-364. https://doi.org/10.1016/j.scrip tamat.2007.04.027

11. Tanaka Y, Kubota A, Matsusaki M, Duncan TJ, Hatakeyama Y, Fukuyama K, Quantock AJ, Yamato M, Akashi M, Nishida K (2011) Anisotropic mechanical properties of collagen hydrogels induced by uniaxial-flow for ocular applications. J Biomater Sci Polym Ed 22:1427-1442. https://doi.org/10.1163/092050610X 510542

12. Bonewald LF (2011) The amazing osteocyte. J Bone Miner Res 26:229-238. https://doi.org/10.1002/jbmr.320

13. Shiraishi A, Miyabe S, Nakano T, Umakoshi Y, Ito M, Mihara M (2009) The combination therapy with alfacalcidol and risedronate improves the mechanical property in lumbar spine by affecting the material properties in an ovariectomized rat model of osteoporosis. BMC Musculoskelet Disord 10:paper \#66. https://doi. org/10.1186/1471-2474-10-66

14. Hara T, Sato T, Oka M, Mori S, Shirai H (2001) Effects of ovariectomy and/or dietary calcium deficiency on bone dynamics in the rat hard palate, mandible and proximal tibia. Arch Oral Biol 46:443-451. https://doi.org/10.1016/S0003-9969(00)00135-7

15. Iwamoto J, Yeh JK, Takeda T, Ichimura S, Sato Y (2003) Comparative effects of vitamin $\mathrm{K}$ and vitamin $\mathrm{D}$ supplementation on prevention of osteopenia in calcium-deficient young rats. Bone 33:557-566. https://doi.org/10.1016/S8756-3282(03)00249-7

16. Shen V, Birchman R, Xu R, Lindsay R, Dempster DW (1995) Short-term changes in histomorphometric and biochemical turnover markers and bone mineral density in estrogen-and/or dietary calcium-deficient rats. Bone 16:149-156. https://doi. org/10.1016/8756-3282(95)80026-M

17. Ke HZ, Jee WSS, Zeng QQ, Li M, Lin BY (1993) Prostaglandin E2 increased rat cortical bone mass when administered immediately following ovariectomy. Bone Mineral 21:189-201. https ://doi.org/10.1016/S0169-6009(08)80230-9

18. Kayanja MM, Ferrara LA, Lieberman IH (2004) Distribution of anterior cortical shear strain after a thoracic wedge compression fracture. Spine J 4:76-87. https://doi.org/10.1016/j.spine e.2003.07.003

19. Schiessl H, Feretti JL, Tysarczyk-Niemeyer G, Willnecker J (1996) Noninvasive bone strength index as analyzed by peripheral quantitative computed tomography (pQCT). In: Schoenau E (ed) Paediatric osteology: new developments in diagnostics and therapy. Elsevier Science, Amsterdam, pp 141-146

20. Sekita A, Matsugaki A, Ishimoto T, Nakano T (2017) Synchronous disruption of anisotropic arrangement of the osteocyte network and collagen/apatite in melanoma bone metastasis. J Struct Biol 197:260-270. https://doi.org/10.1016/j.jsb.2016.12.003

21. Umeno A, Kotani H, Iwasaka M, Ueno S (2001) Quantification of adherent cell orientation and morphology under strong magnetic fields. IEEE Trans Magn 37:2909-2911. https://doi. org/10.1109/20.951344

22. Ishimoto T, Nakano T, Yamamoto M, Tabata Y (2011) Biomechanical evaluation of regenerating long bone by nanoindentation. J Mater Sci Mater Med 22:969-976. https://doi. org/10.1007/s10856-011-4266-y

23. Oliver WC, Pharr GM (1992) An improved technique for determining hardness and elastic modulus using load and displacement sensing indentation experiments. J Mater Res 7:15641583. https://doi.org/10.1557/JMR.1992.1564
24. Bentolila V, Boyce TM, Fyhrie DP, Drumb R, Skerry TM, Schaffler MB (1998) Intracortical remodeling in adult rat long bones after fatigue loading. Bone 23:275-281. https://doi. org/10.1016/S8756-3282(98)00104-5

25. Gluhak-Heinrich J, Pavlin D, Yang W, MacDougall M, Harris SE (2007) MEPE expression in osteocytes during orthodontic tooth movement. Arch Oral Biol 52:684-690. https://doi. org/10.1016/j.archoralbio.2006.12.010

26. Gluhak-Heinrich J, Ye L, Bonewald LF, Feng JQ, MacDougall M, Harris SE, Pavlin D (2003) Mechanical loading stimulates dentin matrix protein 1 (DMP1) expression in osteocytes in vivo. J Bone Miner Res 18:807-817. https://doi.org/10.1359/ jbmr.2003.18.5.807

27. Nampei A, Hashimoto J, Hayashida K, Tsuboi H, Shi K, Tsuji I, Miyashita H, Yamada T, Matsukawa N, Matsumoto M, Morimoto S, Ogihara T, Ochi T, Yoshikawa H (2004) Matrix extracellular phosphoglycoprotein (MEPE) is highly expressed in osteocytes in human bone. J Bone Miner Metab 22:176-184. https://doi.org/10.1007/s00774-003-0468-9

28. Hirao M, Hashimoto J, Yamasaki N, Ando W, Tsuboi H, Myoui A, Yoshikawa H (2007) Oxygen tension is an important mediator of the transformation of osteoblasts to osteocytes. J Bone Miner Metab 25:266-276. https://doi.org/10.1007/s0077 4-007-0765-9

29. O'Brien RM (2007) A caution regarding rules of thumb for variance inflation factors. Qual Quant 41:673-690. https://doi. org/10.1007/s11135-006-9018-6

30. Lei Z, Xiaoying Z, Xingguo L (2009) Ovariectomy-associated changes in bone mineral density and bone marrow haematopoiesis in rats. Int J Exp Pathol 90:512-519. https://doi.org/10.111 1/j.1365-2613.2009.00661.x

31. Tanaka M, Matsugaki A, Ishimoto T, Nakano T (2016) Evaluation of crystallographic orientation of biological apatite at vertebral cortical bone in ovariectomized cynomolgus monkey treated with minodronic acid and alendronate. J Bone Miner Metab 34:234-241. https://doi.org/10.1007/s00774-015-0658-2

32. Chu LLH, Macgregor RR, Anast CS, Hamilton JW, Cohn DV (1973) Studies on the biosynthesis of rat parathyroid hormone and proparathyroid hormone: adaptation of the parathyroid gland to dietary restriction of calcium. Endocrinology 93:915924. https://doi.org/10.1210/endo-93-4-915

33. Divieti P, Inomata N, Chapin K, Singh R, Jüppner H, Bringhurst FR (2001) Receptors for the carboxyl-terminal region of PTH(1-84) are highly expressed in osteocytic cells. Endocrinology 142:916-925. https://doi.org/10.1210/endo.142.2.7955

34. Miyagawa K, Yamazaki M, Kawai M, Nishino J, Koshimizu T, Ohata Y, Tachikawa K, Mikuni-Takagaki Y, Kogo M, Ozono K, Michigami T (2014) Dysregulated gene expression in the primary osteoblasts and osteocytes isolated from hypophosphatemic Hyp mice. PLoS ONE 9:e93840. https://doi. org/10.1371/journal.pone.0093840

35. Pettifor JM, Marie PJ, Sly MR, du Bruyn DB, Ross F, Isdale JM, de Klerk WA, van der Walt WH (1984) The effect of differing dietary calcium and phosphorus contents on mineral metabolism and bone histomorphometry in young vitamin D-replete baboons. Calcif Tissue Int 36:668-676. https://doi.org/10.1007/ BF02405388

36. Kemi VE, Kärkkäinen MU, Rita MJ, Laaksonen MM, Outila TA, Lamberg-Allardt CJ (2010) Low calcium: phosphorus ratio in habitual diets affects serum parathyroid hormone concentration and calcium metabolism in healthy women with adequate calcium intake. Br J Nutr 103:561-568. https://doi.org/10.1017/ S000711450999121

37. Chavassieux P, Seeman E, Delmas PD (2007) Insights into material and structural basis of bone fragility from diseases associated with fractures: how determinants of the biomechanical 
properties of bone are compromised by disease. Endocr Rev 28:151-164. https://doi.org/10.1210/er.2006-0029

38. Peng ZQ, Tuukkanen J, Zhang H, Väänänen HK (1999) Alteration in the mechanical competence and structural properties in the femoral neck and vertebrae of ovariectomized rats. J Bone Miner Res 14:616-623. https://doi.org/10.1359/ jbmr.1999.14.4.616

39. Shahnazari M, Yao W, Dai W, Wang B, Ionova-Martin SS, Ritchie RO, Heeren D, Burghardt AJ, Nicolella DP, Kimiecik MG, Lane NE (2010) Higher doses of bisphosphonates further improve bone mass, architecture, and strength but not the tissue material properties in aged rats. Bone 46:1267-1274. https:// doi.org/10.1016/j.bone.2009.11.019

40. Ammann P, Rizzoli R (2003) Bone strength and its determinants. Osteoporos Int 14 (Suppl 3):S13-S18. https://doi. org/10.1007/s00198-002-1345-4
41. Mashiba T, Mori S, Komatsubara S, Cao Y, Manabe T, Norimatsu H, Burr DB (2005) The effects of suppressed bone remodeling by bisphosphonates on microdamage accumulation and degree of mineralization in the cortical bone of dog rib. J Bone Miner Metab 23:S36-S42

42. Boskey A (2003) Bone mineral crystal size. Osteoporos Int 14:S16-S21. https://doi.org/10.1007/s00198-003-1468-2

43. Saito M, Marumo K (2015) Effects of collagen crosslinking on bone material properties in health and disease. Calcif Tissue Int 97:242-261. https://doi.org/10.1007/s00223-015-9985-5

Publisher's Note Springer Nature remains neutral with regard to jurisdictional claims in published maps and institutional affiliations. 